\title{
Comparación de laparoscopia y boroscopia en la inseminación intrauterina de ovejas
}

\author{
Comparison of laparoscopy and borescopy in the intrauterine \\ insemination of ewes
}

\begin{abstract}
$\searrow$
Jaime Jorge Martínez-Tinajero*凶, Daniel Cruz-Zavala*, Alejandro Muñoz-Bedoya**, Francisco Indalecio Juárez-Lagunes***, Miguel Arcángel Rodríguez-Chessani*****

Martínez-Tinajero, J. J., Cruz-Zavala, D., Muñoz-Bedoya, A., Juárez-Lagunes, F. I., \& Rodríguez-Chessani, M. A. (2019). Comparación de laparoscopia y boroscopia en la inseminación intrauterina de ovejas. Investigación y Ciencia de la Universidad Autónoma de Aguascalientes, 27(76), 89-92.
\end{abstract}

\section{RESUMEN}

Se comparó la técnica de inseminación artificial intrauterina sustituyendo el laparoscopio por un boroscopio y su análisis económico. Se utilizaron 36 ovejas Blackbelly que fueron asignadas a dos grupos ( $A=$ boroscopio, $B=$ laparoscopio). Los estros fueron sincronizados con esponjas impregnadas con MPA más una inyección de eCG después de remover las esponjas. Las variables de estudio fueron inicio del estro, porcentaje de estros, distribución de estros y porcentaje de gestación. El inicio de estro fue 32.50 \pm 7.07 y $34.20 \pm 8.64 \mathrm{~h}$ para grupo A y B, no existiendo diferencias $(P>0.05)$. La presentación de estros fue $100 \%$ para ambas técnicas no existiendo diferencias $(P>0.05)$. El porcentaje de gestación fue de $83.33 \%$ y

Palabras clave: biotecnología; laparoscopia; boroscopio; reproducción; ovejas.

Keywords: biotechnology; laparoscopy; borescope; reproduction; ewes.

Recibido: 16 de noviembre de 2017, aceptado: 14 de agosto de 2018

* Facultad de Ciencias Agrícolas, Campus IV, Universidad Autónoma de Chiapas. Carretera Costera entronque Huehuetán Pueblo, Huehuetán C. P. 30660, Chiapas, México. Correo electrónico: jaimej@unach.mx; danielczavala@hotmail.com. ORCID: orcid.org/0000-0001-9829-9511; orcid.org/0000-0002-3560-6550

** Facultad de Medicina Veterinaria, Universidad de la Amazonia. \#4-1 a, Cl. 17 \#4451, Florencia, Caquetá, Colombia. Correo electrónico: alejo. mube@hotmail.com. ORCID: orcid.org/0000-0002-6216-7717

*** Facultad de Medicina Veterinaria y Zootecnia, Universidad Veracruzana. Circunvalación y Yañez S/N Col. Unidad Veracruzana, C. P. 91710, Veracruz, Veracruz, México. Correo electrónico: fjuarez@ Uv.mx. ORCID: orcid.org/0000-0003-0652-7463

**** Facultad de Medicina Veterinaria y Zootecnia, Universidad Autónoma de Chiapas. Carretera a Emiliano Zapata Km. 8, Tuxtla Gutiérrez, C. P. 29060, Chiapas, México. Correo electrónico: mvelascoz@yahoo.com. $\mathrm{mx}$. ORCID: orcid.org/0000-0003-1605-1067

$凶$ Autor para correspondencia
$77.77 \%$ para A y B no existiendo diferencias $(P>0.05)$. El uso de un boroscopio en sustitución del laparoscopio no afecta el porcentaje de gestación en ovejas y además reduce los costos para su aplicación.

ABSTRACT

The intrauterine artificial insemination technique was compared by replacing the laparoscope with a borescope and its economic analysis. 36 Blackbelly sheep were used that were assigned to two groups ( $\mathrm{A}=$ borescope, $\mathrm{B}=$ laparoscope). Estrus were synchronized with sponges impregnated with MPA plus a shot of eCG after sponge's removal. The study variables were onset of estrus, percentage of estrus presentation and pregnancy percentage. The onset of estrus was $32.50 \pm 7.07$ and $34.20 \pm 8.64$ $\mathrm{h}$ for group $A$ and $B$, respectively, without finding significant differences $(P>0.05)$. The percentage of estrus presentation was $100 \%$ for both techniques, with no significant differences $(P>0.05)$. The pregnancy percentage was 83.33 and $77.77 \%$ for groups $A$ and $B$, and no differences were found $(P>0.05)$. The use of a borescope to replace the laparoscope does not affect the percentage of gestation in sheep and besides reduces the costs for its application.

\section{INTRODUCCIÓN}

La producción de ovinos en México ha evolucionado considerablemente debido al empleo de tecnologías de reproducción y mejoramiento genético, donde es posible encontrar IA por laparoscopia con semen congelado, la cual ha incrementado la productividad en los rebaños al aumentar el índice de concepción a 78.57\% (Godfrey, Gray, \& Collins, 1998). Este método 
consiste en la aplicación de semen directamente dentro del lumen uterino, evitando la barrera natural del cérvix. Dogan, Nur, Gunay, Soylu y Sonmez (2004) mencionan que la IA por laparoscopia es una técnica que emplea una cirugía menor para depositar directamente semen congelado dentro de la cavidad uterina. Su implementación ha generado resultados satisfactorios, ya que permite el uso de sementales de alta calidad genética, especialmente por la utilización de semen congelado, que resulta ser la selección más acertada. Hidalgo et al. (2015) mencionan que la inseminación artificial por laparoscopia con semen congelado se puede realizar a tiempo fijo superando los resultados de la inseminación artificial pericervical, cervical y transcervical. Sin embargo, esta se limita a un grupo reducido de productores, debido al alto costo de su implementación. En 1945 el Dr. Karl Storz empezó a fabricar instrumentos de otorrinolaringología con la intención de crear sistemas y dispositivos que le permitieran observar dentro del cuerpo humano, consistente en dirigir a través de un instrumento una luz clara pero fría hasta las cavidades corporales y de ese modo facilitar una visión nítida; al mismo tiempo, hacer una documentación objetiva mediante la transferencia de imágenes. Los boroscopios son resistentes y han sido diseñados para la industria, ya que constan de un sistema de lentes cilíndricos, lo que pronuncia imágenes luminosas y nítidas. Por lo anterior, el objetivo del presente estudio fue utilizar un boroscopio industrial en sustitución del laparoscopio durante el protocolo de inseminación artificial intrauterina para reducir el costo de dicha técnica.

\section{MATERIALES Y MÉTODOS}

El estudio se realizó en el otoño de 2017 en el Centro de Mejoramiento Genético Granja Los Martínez, ubicado entre los $14^{\circ} 46^{\prime} \mathrm{N}$ y $92^{\circ} 18^{\prime} \mathrm{O}$ y localizado en el municipio de Tapachula, Chiapas, México. Se utilizaron 36 ovejas Blackbelly de segundo parto con $26.2 \pm 2.7$ meses de edad y $34.6 \pm 2.9 \mathrm{~kg}$ de peso corporal, manejadas en un sistema de libre pastoreo con distribución completamente al azar en dos grupos experimentales: ( $A=$ boroscopia, $n=18)$ y $(B=$ laparoscopia, $n=18)$. Las ovejas se sometieron a un protocolo de sincronización de estros mediante esponjas intravaginales impregnadas con $60 \mathrm{mg}$ de acetato de medroxiprogesterona (MPA) durante 12 d más una inyección intramuscular de $250 \mathrm{UI}$ de gonadotropina coriónica equina (eCG) al retiro de las esponjas. El estro se detectó con un macho marcador a través de montas falsas. La recolección de semen se realizó en la mañana del día de la inseminación. El semen se recolectó por medio de vagina artificial e inmediatamente se evaluó la calidad seminal (volumen, color, motilidad y concentración espermática). La dilución del semen se realizó con Triladyl (20\%), agua bidestilada (60\%) y yema de huevo (20\%), con la finalidad de obtener $90 \mathrm{M}$ de espermatozoides por dosis de semen en pajillas de $0.25 \mathrm{ml}$. Una vez diluido se mantuvo a una temperatura de $37^{\circ} \mathrm{C}$ hasta su aplicación. Las hembras fueroninseminadas a tiempo fijo 48 h después de retiradas las esponjas evaluando las variables, inicio de estro, distribución y porcentaje del mismo y porcentaje de gestación. Para determinar la variable de porcentaje de gestación se realizó un diagnóstico de gestación a los 45 d después de la inseminación artificial, en donde se utilizó un ultrasonido Sonoace 600 (Medison) con un transductor transabdominal de $7.5 \mathrm{MHz}$. Las variables continuas fueron estimadas con el análisis de varianza y las de proporción con la prueba no paramétrica Chi cuadrada (Herrera Haro, \& Barreras Serrano, 2005).

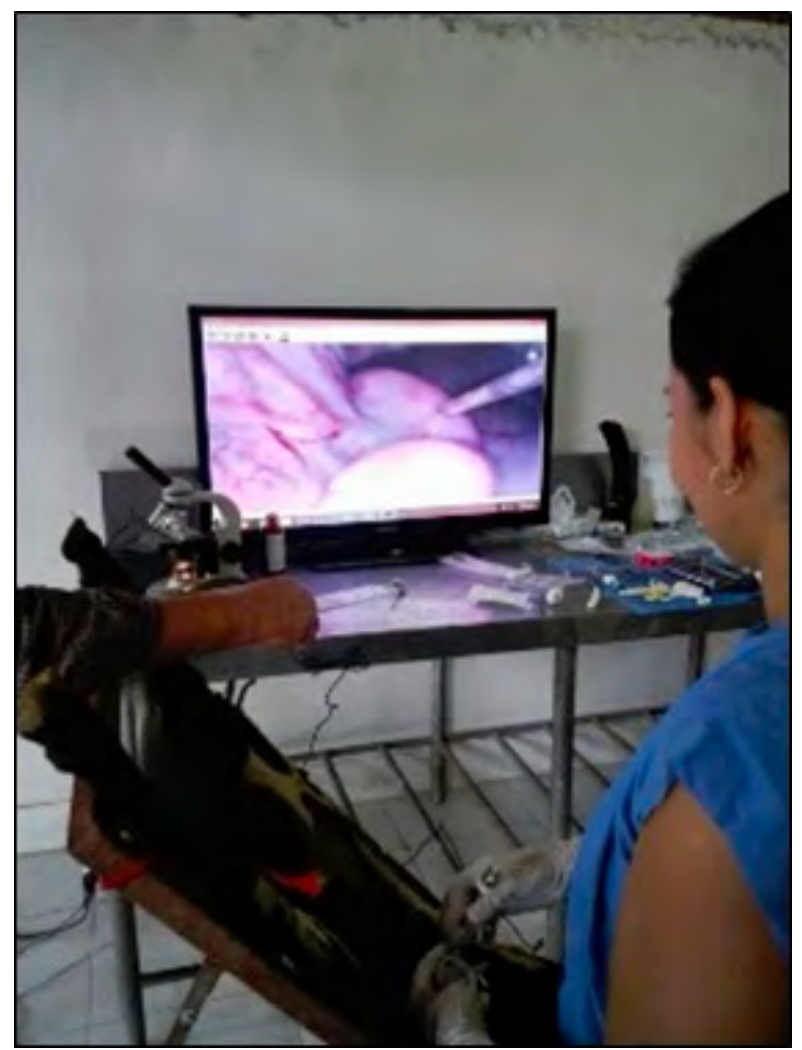

Figura 1. Se estudia la inseminación artificial intrauterina sustituyendo el laparoscopio por un boroscopio y su análisis económico.

Fotografía tomada por el equipo de investigación. 
Tabla 1

Respuesta reproductiva de ovejas inseminadas por laparoscopia y usando boroscopio sincronizadas con esponjas MPA. $250 \mathrm{UI}$ eCG

\begin{tabular}{ccccc}
\hline Tratamientos & $\mathrm{N}$ & Inicio de estro $(\mathrm{h})$ & Presentación de estro (\%) & Gestación (\%) \\
\hline Grupo A & 18 & $32.50 \pm 7.07_{a}$ & $100_{a}$ & $83.33_{a}$ \\
\hline Grupo B & 18 & $34.20 \pm 8.64_{a}$ & $100_{a}$ & $77.77_{a}$ \\
\hline Total & 36 & $33.35 \pm 7.56$ & & \\
\hline
\end{tabular}

Nota: a Misma literal significa que no existieron diferencias estadísticas.

Elaboración propia.

\section{RESULTADOS}

El tiempo promedio del inicio de estro fue de $33.35 \pm$ $7.56 \mathrm{~h}$ después del retiro de las esponjas sin diferencias $(P>0.05)$ entre grupos. Los valores promedio del inicio del estro fueron $32.50 \pm 7.07$ y $34.20 \pm 8.64 \mathrm{~h}$ para los grupos A y B, respectivamente. Estos datos son similares a lo reportado por González-Reyna, LuceroMagaña, Briones-Encinia, Limas-Martínez y MartínezGonzález (2014), quienes registraron inicios de estro de 28.6 a 34.6 h después del retiro de las esponjas. La totalidad de las ovejas presentaron estro después del tratamiento con esponjas sin encontrar diferencias $(P>0.05)$ (tabla 1). Estos datos coinciden con lo reportado por Martínez-Tinajero et al. (2007), donde señalan que las ovejas sincronizadas con MAP + eCG presentan iguales resultados (100\%).

El porcentaje de gestación fue de 83.33 y $77.77 \%$ para las ovejas de los grupos experimentales A y $B$, sin diferencias $(P>0.05)$ entre ambas técnicas. Con el uso del laparoscopio se observa una buena imagen de los cuernos uterinos pero es necesario estar limpiando el telescopio y cuidando el cable de fibra óptica para evitar accidentes que afecten el equipo; mientras tanto, el uso del boroscopio es más factible y menos delicado, ya que se puede adaptar a una computadora, televisión y/o teléfono inteligente para visualizar la imagen del cuerno uterino, lo que permite obtener mejores resultados en el porcentaje de gestación. No se ha encontrado literatura científica en el uso del boroscopio industrial en la inseminación artificial intrauterina. Sin embargo, la técnica de inseminación por laparoscopia es cara por los materiales a utilizar, por lo que una alternativa para reducir costos es sustituir el equipo de laparoscopia por un boroscopio, que permite reducir $98 \%$ de los costos.

CONCLUSIONES

El uso de un boroscopio industrial en sustitución del laparoscopio no tiene efecto significativo sobre el porcentaje de gestación en ovejas sincronizadas al estro e inseminadas con semen fresco y además reduce los costos para su aplicación. 


\section{REFERENCIAS}

- Dogan, I., Nur, Z., Gunay, U., Soylu, M. K., \& Sonmez, C. (2004). Comparison of fluorgestone and medroxyprogesterone intravaginal sponges for estrus synchronization in Saanen does during the transition period. South African Journal of Animal Science, 34(1), 18-22.

- Godfrey, R. W., Gray, M. L., \& Collins, J. R. (1998). The effect of ram exposure on uterine involution and luteal function during the postpartum period of hair sheep ewes in the tropics. Journal of Animal Science, 76, 3090-3094. Recuperado de https://pdfs.semanticscholar.org/4538/8908776b5e48b88c20 dc94d290a3678b8bc5.pdf

- González-Reyna, A., Lucero-Magaña, F. A., Briones-Encinia, F., Limas-Martínez, G., \& Martínez-González, J. C. (2014). Factores que alteran la conducta en ovejas de pelo sincronizadas con acetato de fluorogesterona y gonadotropina de suero de yegua preñada. Abanico Veterinario, 4(2), 13-20.

- Herrera Haro, J. G., \& Barreras Serrano, A. (2005). Manual de procedimientos: Análisis estadísticos de experimentos pecuarios: utilizando el programa SAS (3a. ed.). México, D. F.: Colegio de Postgraduados.

- Martínez-Tinajero, J. J., Izaguirre Flores, F., Sánchez Orozco, L., García Castillo, C. G., Martínez-Priego, G., \& Torres Hernández, G. (2007). Comportamiento reproductivo de ovejas Barbados barriga negra sincronizadas con MPA y diferentes tiempos de aplicación de eCG durante la época de baja fertilidad. Revista Científica, FCV-LUZ, 17(1), 47-52. 